\title{
Extensive neurological recovery from a complete spinal cord injury: a case report and hypothesis on the role of cortical plasticity
}

\section{Ann S. Choe ${ }^{1,2,3 \dagger}$, Visar Belegu ${ }^{1,2+}$, Shoko Yoshida ${ }^{4}$, Suresh Joel ${ }^{3,4}$, Cristina L. Sadowsky ${ }^{2,5}$, Seth A. Smith ${ }^{6}$, Peter C. M. van Zijli, ${ }^{3,4}$, James J. Pekar ${ }^{3,4}$ and John W. McDonald ${ }^{1,2,5 *}$}

${ }^{1}$ Department of Neurology, Johns Hopkins University School of Medicine, Baltimore, MD, USA

2 International Center for Spinal Cord Injury, Hugo W. Moser Research Institute at Kennedy Krieger, Inc., Baltimore, MD, USA

${ }^{3}$ F. M. Kirby Research Center for Functional Brain Imaging, Kennedy Krieger Institute, Baltimore, MD, USA

${ }^{4}$ Russell H. Morgan Department of Radiology and Radiological Science, Johns Hopkins University School of Medicine, Baltimore, MD, USA

${ }^{5}$ Physical Medicine and Rehabilitation, Johns Hopkins University School of Medicine, Baltimore, MD, USA

${ }^{6}$ Department of Radiology and Radiological Science, Institute of Imaging Science, Vanderbilt University, Nashville, TN, USA

\section{Edited by:}

Sven Bestmann, University College London, UK

\section{Reviewed by:}

Patrick Freund, University College London Institute of Neurology, UK Antonio Oliviero, Hospital Nacional de Paraplejicos, Spain

\section{*Correspondence:}

John W. McDonald, Kennedy

Krieger Institute, Johns Hopkins

University School of Medicine, 707

N. Broadway, Baltimore, MD 21205,

USA

e-mail:mcdonaldj@

kennedykrieger.org

these authors have contributed equally to this work.
Neurological recovery in patients with severe spinal cord injury (SCI) is extremely rare. We have identified a patient with chronic cervical traumatic $\mathrm{SCl}$, who suffered a complete loss of motor and sensory function below the injury for 6 weeks after the injury, but experienced a progressive neurological recovery that continued for 17 years. The extent of the patient's recovery from the severe trauma-induced paralysis is rare and remarkable. A detailed study of this patient using diffusion tensor imaging (DTI), magnetization transfer imaging (MTI), and resting state fMRI (rs-fMRI) revealed structural and functional changes in the central nervous system that may be associated with the neurological recovery. Sixty-two percent cervical cord white matter atrophy was observed. DTI-derived quantities, more sensitive to axons, demonstrated focal changes, while MTI-derived quantity, more sensitive to myelin, showed a diffuse change. No significant cortical structural changes were observed, while rs-fMRI revealed increased brain functional connectivity between sensorimotor and visual networks. The study provides comprehensive description of the structural and functional changes in the patient using advanced MR imaging technique. This multimodal MR imaging study also shows the potential of rs-fMRI to measure the extent of cortical plasticity.

Keywords: spinal cord injury, trauma, diffusion tensor imaging, magnetization transfer imaging, resting state fMRI, plasticity

\section{CASE PRESENTATION}

The patient, a 58-year-old male, endured vertebral fractures at cervical levels $\mathrm{C} 3-\mathrm{C} 6$ in a motor vehicle accident at the age of 22. The patient did not have any respiratory problem and his stay in the ICU was limited to 5 days. Following the injury, the patient underwent manual motor testing of lower and upper body muscles groups as well as sensory testing. The motor testing involved examination of flexion and extension of upper body (shoulder, elbow, and wrist), and lower body joints (hip, knee, and ankle), and sensory testing involved examination of sensation to pinprick and light touch on both hands and feet. Initially, the patient had no motor or sensory function below the level of injury. Motor and sensory function at the sacral level was also absent, which was tested during the bladder evacuation and catheter placement procedure. This injury is equivalent to an ASIA A (ASIA: American Spinal Injury Association), or a "complete" injury, according to today's ASIA impairment scale (AIS) (Maynard et al., 1997), which did not exist at the time of the patient's injury.

Spinal cord stabilization was accomplished with a halo. His rehabilitation regimen consisted of active-assisted range of motion (ROM) exercises of the lower and upper limb performed at home. Lower limb ROM exercise was performed for $30 \mathrm{~min}$, three times a day, and the upper limb ROM exercise was performed for $30 \mathrm{~min}$, two times a day. The exercise was performed at home, assisted by his family members.

$\mathrm{He}$ experienced slow and progressive neurological recovery that continued for 17 years after the injury. The first neurological recovery, movement of the left big toe (a grade 1 motor function), occurred 6 weeks after the injury. The first recovery of sensation occurred 6 months after the injury in the form of a painful dysesthetic sensation in the pelvis. Eleven months after the injury, the patient was able to perform complex upper body motor functions such as sitting up without assistance. Complex lower body motor functions such as ability to walk unassisted were recovered 15 months after the injury. Concomitantly, the patient recovered autonomic nervous system functions (i.e., a high degree of bladder and bowel functions were recovered up to 64 months after the injury) although many remain abnormal (Table 1). Currently, his injury is categorized as ASIA D. His latest AIS evaluation (Figure 1) showed that he has regained $94 \%$ of motor function in the upper body and 100\% in the lower body. 
Table 1 | Detailed timeline of the patient's recovery.

\begin{tabular}{|c|c|c|}
\hline & Date & Description \\
\hline 0 & Sep-74 & Accident \\
\hline 1 & Nov-74 & $\begin{array}{l}\text { Flicker of contraction present at left big toe. No sensory } \\
\text { recovery }\end{array}$ \\
\hline 2 & Mar-75 & Flicker of contraction present at left quadriceps \\
\hline 3 & Mar-75 & Dysesthetic (abnormal) sensation at pelvis \\
\hline 4 & Mar-75 & Flick of sensation present at left bicep \\
\hline 5 & Mar-75 & Flick of sensation present at left fingers \\
\hline 6 & Apr-75 & Flicker of contraction present at right big toe \\
\hline 7 & May-75 & Begin to gain trunk movement \\
\hline 8 & May-75 & Begin working on trunk balance \\
\hline 9 & Jun-75 & Sit with $50 \%$ assistance \\
\hline 10 & Jun-75 & Flicker of contraction present at left triceps \\
\hline 11 & Oct-75 & Sit without assistance \\
\hline 12 & Dec-75 & Active movement of left arm against gravity \\
\hline 13 & Jan-76 & Stand with $50 \%$ assistance \\
\hline 14 & Feb-76 & Regain ability to grip with hands \\
\hline 15 & May-76 & Regained ability to ejaculate \\
\hline 16 & Jun-76 & Stand without assistance \\
\hline 17 & Sep-76 & First swim \\
\hline 18 & Jan-77 & Begin to gain control of bladder movement (incomplete) \\
\hline 19 & Jan-78 & First walk 40 feet without assistance \\
\hline 20 & Jun-78 & Could walk in community \\
\hline 21 & Jan-79 & Begin to gain control of bowel movements (incomplete) \\
\hline 22 & $1978-1980$ & Functional recovery rate begins to plateau \\
\hline 23 & Apr-91 & First run \\
\hline
\end{tabular}

The 1974 motor accident [0] resulted in a complete loss of motor and sensory function below the injury epicenter that lasted 6 weeks after the injury. Initial regaining of motor and sensory function [1,3] was then followed by a long but steady recovery process that continued for 17 years after the injury, where at the end of the 17 years, he was able to run without assistance [23].

The patient manifests incomplete and asymmetric motor recovery in his hands-i.e., his left hand is more functional then the right one. He has also regained $23 \%$ of sensory function in the upper body (above the level of T7), but only 10\% in the lower body. The patient reports that his walking and functions he performs with his feet depend heavily on visual feedback; he also manifests autonomic dysreflexia. It should be noted that the AIS evaluation of the motor function is based on muscle strength in the extremities, and that sensory function is evaluated based on conscious sensation of light touch and pin prick on the skin, and does not include proprioception.

\section{BACKGROUND}

The spinal cord is a conduit for the exchange of information between the brain and the body, and damage to it disrupts conduction of sensory and motor signals across the lesion epicenter (Belegu et al., 2007). The extent of the neurological impairment following spinal cord injury (SCI) varies, and the severity of the injury limits the subsequent neurological recovery $(\mathrm{McD}$ onald and Sadowsky, 2002; Kirshblum et al., 2004). Severely injured SCI patients, classified as ASIA A on the AIS (Maynard et al., 1997), experience limited neurological recovery that often does not translate to functional improvements. Patients with less severe injuries, classified as ASIA B-D, experience greater recovery (Kirshblum et al., 2004). This is further corroborated by a retrospective study of 173 traumatic SCI patients with 5-year follow up (Vazquez et al., 2008), which reported relatively good recovery results for patients with incomplete injury; at the time of their discharge, $33.3 \%$ the ASIA B patients, $76.4 \%$ of the ASIA C patients, and $100 \%$ of the ASIA D patients showed some degree of neurological recovery. The same study also reported, however, that $94 \%$ of the ASIA A patients remained complete upon discharge without any neurological recovery and none of them were functional at the time of their discharge (Vazquez et al., 2008). Chances of neurological recovery for ASIA A patients become even smaller in chronic phase of SCI (McDonald and Sadowsky, 2002).

Two variables that contribute to the capacity for neurological recovery are well understood. First, preservation of longitudinally oriented axonal tracts in the white matter is required (Blight, 1983; Nathan, 1994). Second, proper conductance through the spared white matter axons and hence proper myelination is required. However, only $5 \%$ of intact axons in the white matter tracts are needed to procure locomotion following SCI in cats (Blight, 1983), while in humans, locomotion can recover after resection of $25 \%$ of the spinal cord (Nathan, 1994), indicating that preservations of axons and myelination are not proportional to the degree of neurological recovery. Task-activated functional MRI (fMRI) studies show different activation pattern between patients who experience neurological recovery and those who do not (Jurkiewicz et al., 2007, 2010), suggesting that cortical plasticity may be a third variable. While the occurrence of cortical plasticity subsequent to SCI has been observed (Endo et al., 2007; Jurkiewicz et al., 2007, 2010), it is unclear how it leads to neurological recovery.

Here, we present a report of a patient with a chronic cervical traumatic SCI, who suffered a complete loss of motor and sensory function below the injury epicenter for 6 weeks after the injury, and experienced a slow and progressive neurological recovery that continued for 17 years. The extent of the recovery is so remarkable that it represents a pragmatic cure from trauma induced complete paralysis. We utilized advanced MRI technologies such as diffusion tensor imaging (DTI), magnetization transfer imaging (MTI), and resting state fMRI (rs-fMRI) to investigate the patient's late recovery. While DTI and MTI showed abnormalities in the spinal cord that indicate axonal and myelin damage, but are not associated with the patient's late recovery, rs-fMRI provided a picture of cortical plasticity that is associated with the patient's late recovery.

\section{MATERIALS AND METHODS PARTICIPANTS}

Spinal cord DTI, brain DTI, and brain rs-fMRI data of healthy controls were obtained from previously acquired and separate databases that exist in the International Center for Spinal Cord Injury (ICSCI) and F.M. Kirby center. Spinal cord imaging was performed in ten healthy volunteers (21-49 years, mean 33, $\mathrm{M} / \mathrm{F}$ ratio: 6/4). For brain imaging, DTI was performed in six age matched healthy volunteers (55-61 years, mean 59, M/F 


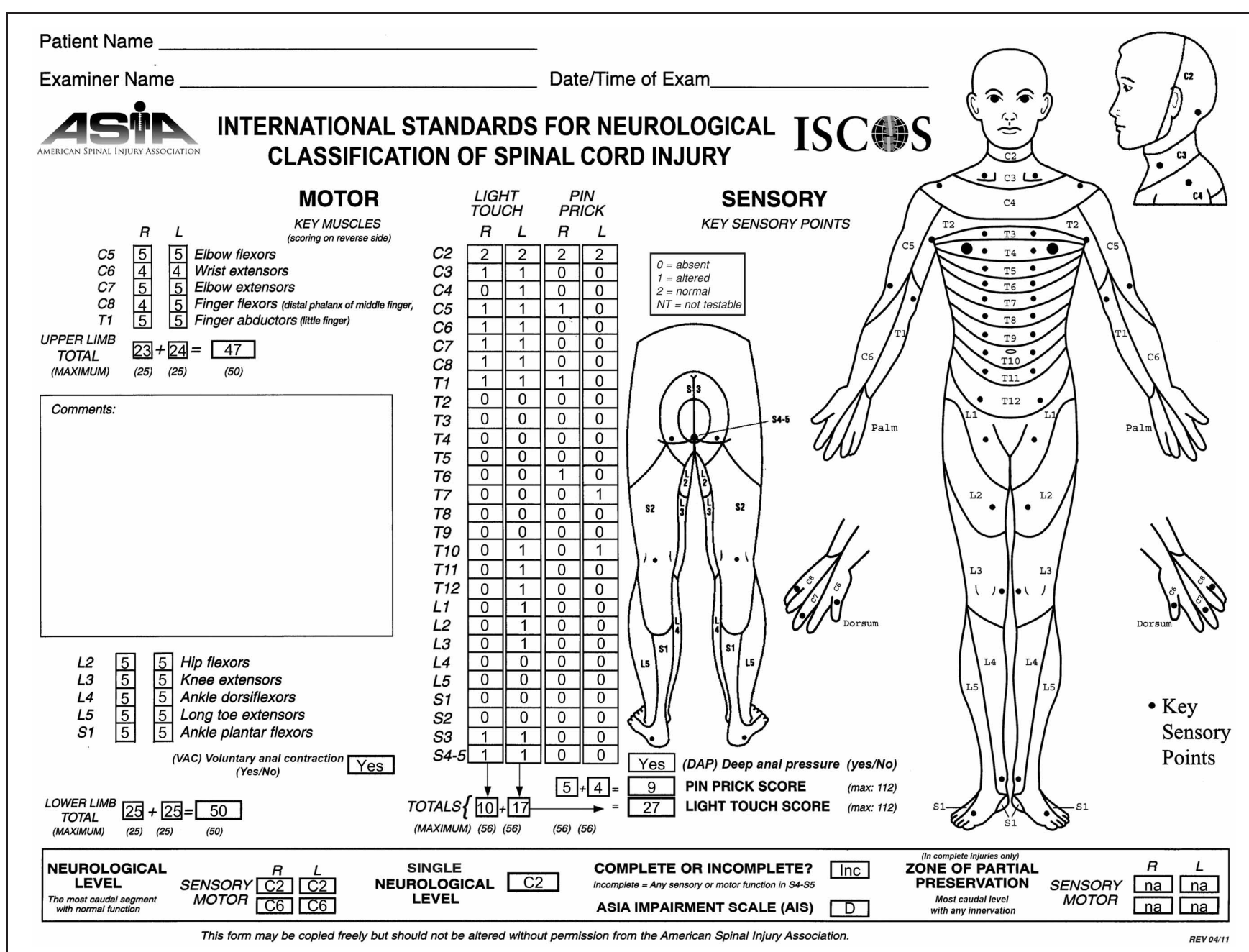

FIGURE 1 | ASIA classification of the patient after recovery, performed according to the International Standards for Neurological Classification.

ratio: 2:4), and rs-fMRI was performed in 21 (22-61 years, mean 32, M/F ratio: 11/10; available on web: www.nitrc.org/ projects/multimodal) healthy volunteers (Landman et al., 2011). Only the six healthy participants from the brain DTI database were age matched due to the limited number of older healthy participants in the spinal cord DTI and brain rs-fMRI databases. All participants gave their informed consent, and the study protocol was approved by the institutional review board at Johns Hopkins University.

\section{IMAGE ACQUISITION}

Participants were scanned on a Philips 3T scanner. The scan room was lit during the scan, but the lights were kept off inside the scanner bore. For spinal cord imaging, DTI was performed using a multi-slice, pulsed gradient spin echo (PGSE) sequence $\left(b=0,500 \mathrm{~s} / \mathrm{mm}^{2}, 16\right.$ diffusion weighting directions, TR/TE $=$ $6300 / 63 \mathrm{~ms}$, SENSE factor $=2,1.5 \times 1.5 \times 3 \mathrm{~mm}^{3}$ original resolution (axial sections of $3 \mathrm{~mm}$ thickness), $0.57 \times 0.57 \times 3 \mathrm{~mm}^{3}$ final resolution (after zero-fill), scan time $=2 \mathrm{~min}$, 16 -channel neurovascular coil) (Figures 2D,H). MT-weighted images were acquired using a three dimensional (3D) spoiled gradient-echo sequence with an EPI readout (EPI factor $=3$, TR/TE $=$ $102 / 13 \mathrm{~ms}, \alpha=9^{\circ}$, and SENSE factor $=2,0.61 \times 0.69 \times 3 \mathrm{~mm}^{3}$ resolution, scan time $=8 \mathrm{~min}, 16$-channel neurovascular coil) (Figures 2C,G). A sagittal T2 weighted (T2-w) was also acquired (Figures 2A,B,E,F). The total scan time for the spinal cord imaging was $28 \mathrm{~min}$.

Brain DTI was performed using a multi-slice PGSE sequence ( $b=0,700 \mathrm{~s} / \mathrm{mm}^{2}, 32$ directions, TR/TE $=7270 / 71 \mathrm{~ms}$, SENSE factor $=2.5,2.2 \times 2.2 \times 2.2 \mathrm{~mm}^{3}$ original resolution, $0.83 \times$ $0.83 \times 2.2 \mathrm{~mm}^{3}$ final resolution, scan time $=5 \mathrm{~min}, 16$-channel neurovascular coil). A high resolution $\mathrm{T} 1$ weighted (T1-w) MPRAGE image $(\mathrm{TR} / \mathrm{TE}=10 / 6 \mathrm{~ms}$, SENSE factor $=2,1.10 \times$ $1.10 \times 1.17 \mathrm{~mm}^{3}$ original resolution, $0.83 \times 0.83 \times 1.17 \mathrm{~mm}^{3}$ final resolution, scan time $=5 \mathrm{~min}$, 16-channel neurovascular coil) was also obtained.

Each rs-fMRI scan of the controls (Landman et al., 2011) was acquired using a single-shot SENSE-EPI (TR/TE $=2000 / 30 \mathrm{~ms}$, SENSE factor $=2,3 \times 3 \times 3 \mathrm{~mm}^{3}$ resolution, $1 \mathrm{~mm}$ gap, scan time $=420 \mathrm{~s}$, 32-channel head coil). Identical imaging parameters 

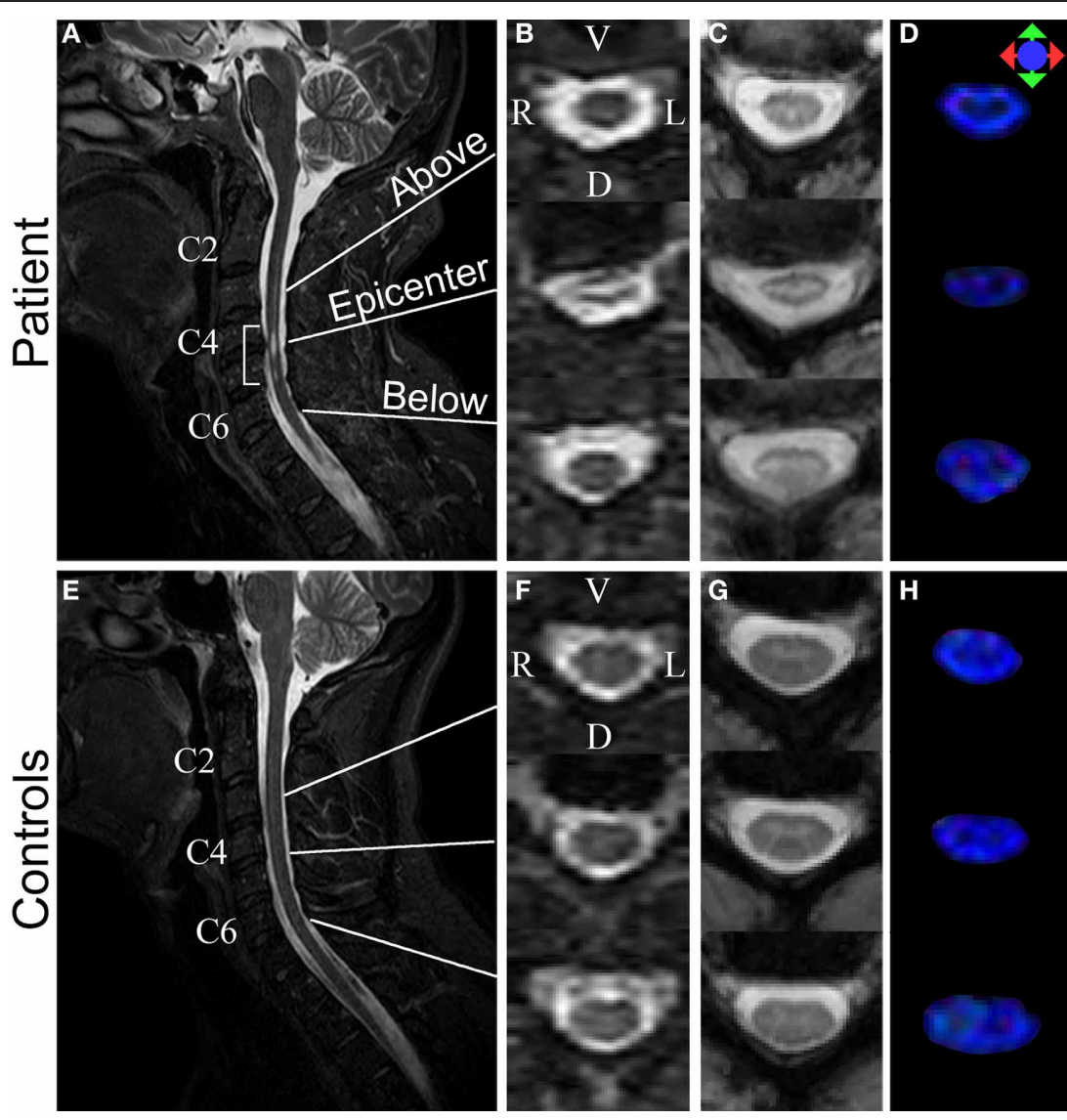

FIGURE 2 | Structural MR images acquired from the patient (top row) and a control (bottom row). (A,E) Sagittal T2-w images. Bracket in (A) highlights injury epicenter of the patient. (B,F) Axial T2-w images. (C,G) Axial MTCSF images. (D,H) Axial FA color maps. The FA maps are color-coded using a standard diffusion color-encoding scheme (r/l: red, a/p: green, s/i: blue). Each row of (B-D) shows a section from: (1) above the injury epicenter, (2) injury epicenter, and (3) below the injury epicenter. Images from the corresponding cervical levels in the control are shown in each row of (F-H). were used to acquire the rs-fMRI data of the SCI patient, with these differences: (1) time of each run: $360 \mathrm{~s}$, (2) number of runs acquired: 8 . For the controls, the first $360 \mathrm{~s}$ of the data was used for further analysis. Total scan time for the brain imaging was $30 \mathrm{~min}$. The rs-fMRI scans were always acquired after the brain T1-w image acquisition to allow the participants to get acclimated to the noise and the new environment inside the scanner. The participants were instructed to stay as still as possible with their eyes closed during the entire scan. A dataset from one of the healthy controls was excluded due to excessive motion.

\section{IMAGE REGISTRATION FOR SPINAL CORD IMAGING}

Basic eddy current correction was performed on the spinal cord and brain DTI datasets using CATNAP (Coregistration, Adjustment, and Tensor-solving, a Nicely Automated Program) (Landman et al., 2007), which performs volume-wise coregistration using a method based on FLIRT (FMRIB's Linear Imaging Registration Tool, Oxford, UK) (Jenkinson et al., 2002). Each diffusion weighted image was registered to an initial $b=0 \mathrm{~s} / \mathrm{mm}^{2}$ (b0, non-diffusion weighted image) volume using a six degrees of freedom, rigid-body registration. Diffusion gradient tables were updated to account for any rotation.
The registration of the MTI dataset to the mean b0 volume was performed in two steps. First, the MTI dataset was registered to the b0 volume using a 3D, six degrees of freedom, rigid body transformation using a similar method proposed by Maes et al. (1999). This 3D registration procedure was then followed by a two-dimensional (2D) registration, using a three degrees of freedom rigid body transformation that involved two in-plane translations and one rotation. The second coregistration procedure was implemented for added accuracy of the result (Smith et al., 2010).

\section{MEASUREMENT OF WHOLE CORD AND WHITE MATTER CROSS-SECTIONAL AREA}

The whole cord and white matter atrophy of the patient's spinal cord was measured using fractional anisotropy (FA) maps. We first separated the spinal cord and the surrounding cerebrospinal fluid (CSF) area from the rest of the neck region by performing manual segmentation. This was done by placing ellipsoidal binary masks around the spinal cord and the surrounding CSF area. Once the segmentation of the spinal cord and CSF was done, the whole cord cross-sectional area (wca) of each slice of the controls and the patient was measured automatically using 
the Otsu's thresholding method, therefore reducing rater bias. The segmented FA maps of the cords were then thresholded at 0.2-the same threshold used during the subsequent fiber tractograophy for the column-specific data analysis - to create the masks of the white matter only area (wma). We have previously shown that FA measures performed in this manner have acceptable inter-rater and test-retest reliability (Smith et al., 2010), and this is expected to extend to the volume measures based on this parameter.

\section{DIFFUSION FIBER TRACTOGRAPHY OF SPINAL COLUMNS AND CREATION OF COLUMN PROFILES}

After the registration of DTI and MTI images and the calculation of DTI- and MTI-derived quantities, diffusion fiber tractography was performed using DTIStudio (Jiang et al., 2006). Higher contrast MTCSF (MT-weighted signal intensity relative to cerebrospinal fluid) images-compared to T2-w images and FA maps-were used to manually draw regions of interest (ROIs) within left and right lateral, dorsal, and ventral columns of the spinal cords. This manual ROI selection was performed on every third section along the entire cervical cord, to be used as seed regions for the fiber tractography to follow. Note that the registered MTCSF images are in the same image space as that of the DTI images. FA threshold of 0.2 and a maximum tract turning angle of $60^{\circ}$ were used as the fiber tractography stopping criterion. Spurious fibers, such as those that cross from one spinal column to the next or move out of the spinal cord, were manually excluded.

Once each spinal column was reconstructed, vertebrae level of C2 and C6 were identified and the corresponding column profiles (Smith et al., 2010; Cohen-Adad et al., 2013) were created. In our previous study (Smith et al., 2010), typical neck length of study participants was found to be $75 \mathrm{~mm}$, which corresponds to $25,3 \mathrm{~mm}$ thickness image sections. Using this information, each column profile was spatially normalized to 25 points spanning C2-C6.

\section{INJURY REGION SPECIFIC DATA ANALYSIS}

Tables 2, 3 report the column profile values of the patient (mean) and the controls (mean \pm SD) for three spinal cord regions: (1) above the injury epicenter, (2) injury epicenter, and (3) below the injury epicenter. The injury epicenter was identified using the patient's T2-w images (Figure 2B). One vertebrae level (approximately $15 \mathrm{~mm}$ ) above the beginning of the lesion site was defined as the region "above" the injury epicenter, and one vertebrae level down from the end of the lesion site was defined as the "below" the injury epicenter. The three spinal cord regions of the controls were defined to match the location of the patient's regions.

Each cell in Table 2 is color coded according to the degree of difference between the values of the controls and patient: (1) cells without color indicate difference less than one SD, (2) light gray cells indicate difference of more than one SD but less than twice the SD, (3) dark gray cells indicate difference of bigger than twice the SD. When DTI and MTI derived parameters were averaged along the whole cord, only FA of the right lateral column and MTCSF of the dorsal column were found to have changes of more
Table 2 | Injury region specific data comparison of the patient.

\begin{tabular}{|c|c|c|c|c|}
\hline & \multicolumn{4}{|c|}{ Patient } \\
\hline & \multicolumn{4}{|c|}{ Mean } \\
\hline & Below & Epicenter & Above & Total \\
\hline \multicolumn{5}{|l|}{ Left lateral } \\
\hline FA & 0.62 & 0.67 & 0.69 & 0.67 \\
\hline $\mathrm{MD}\left(\mu \mathrm{m}^{2} / \mathrm{ms}\right)$ & 1.2 & 1.02 & 0.83 & 1 \\
\hline$\lambda_{\|}\left(\mu \mathrm{m}^{2} / \mathrm{ms}\right)$ & 2.08 & 1.86 & 1.58 & 1.83 \\
\hline$\lambda_{\perp}\left(\mu \mathrm{m}^{2} / \mathrm{ms}\right)$ & 0.76 & 0.62 & 0.47 & 0.6 \\
\hline MTCSF & 0.69 & 0.72 & 0.62 & 0.65 \\
\hline \multicolumn{5}{|l|}{ Right lateral } \\
\hline FA & 0.59 & 0.49 & 0.72 & 0.61 \\
\hline $\mathrm{MD}\left(\mu \mathrm{m}^{2} / \mathrm{ms}\right)$ & 1.03 & 1.40 & 0.79 & 1.14 \\
\hline$\lambda_{\|}\left(\mu \mathrm{m}^{2} / \mathrm{ms}\right)$ & 1.76 & 2.18 & 1.53 & 1.93 \\
\hline$\lambda_{\perp}\left(\mu \mathrm{m}^{2} / \mathrm{ms}\right)$ & 0.66 & 1.01 & 0.43 & 0.76 \\
\hline MTCSF & 0.78 & 0.68 & 0.63 & 0.68 \\
\hline \multicolumn{5}{|l|}{ Dorsal } \\
\hline FA & 0.71 & 0.55 & 0.73 & 0.67 \\
\hline $\mathrm{MD}\left(\mu \mathrm{m}^{2} / \mathrm{ms}\right)$ & 1.13 & 1.40 & 0.97 & 1.14 \\
\hline$\lambda_{\|}\left(\mu \mathrm{m}^{2} / \mathrm{ms}\right)$ & 2.19 & 2.33 & 1.96 & 2.1 \\
\hline$\lambda_{\perp}\left(\mu \mathrm{m}^{2} / \mathrm{ms}\right)$ & 0.6 & 0.94 & 0.48 & 0.66 \\
\hline MTCSF & 0.65 & 0.72 & 0.67 & 0.65 \\
\hline \multicolumn{5}{|l|}{ Ventral } \\
\hline FA & 0.55 & 0.46 & 0.67 & 0.55 \\
\hline $\mathrm{MD}\left(\mu \mathrm{m}^{2} / \mathrm{ms}\right)$ & 1.27 & 1.79 & 0.91 & 1.33 \\
\hline$\lambda_{\|}\left(\mu \mathrm{m}^{2} / \mathrm{ms}\right)$ & 2.06 & 2.61 & 1.67 & 2.12 \\
\hline$\lambda_{\perp}\left(\mu \mathrm{m}^{2} / \mathrm{ms}\right)$ & 0.87 & 1.38 & 0.54 & 0.94 \\
\hline MTCSF & 0.73 & 0.71 & 0.63 & 0.66 \\
\hline
\end{tabular}

Imaging parameters (FA, MD, $\lambda_{\|}, \lambda_{\perp}$, and MTCSF) were measured for the regions of above the injury, injury epicenter, and below the injury.

Values reported are mean of each injury region. Examining the cervical cord in an injury region specific way provides better insight into the actual changes occurring along the patient's cervical spinal cord, as opposed to looking at a single average values of an entire spinal column.

Difference compared to the controls.

Within normal range

Difference is bigger than $1 \times S D$

Difference is bigger than $2 \times S D$

than twice the SD (Table 2). A detailed look at the Tables 2, 3, however, reveals that there are substantial changes of DTI- and MTI-derived parameters above, at, and below the injury epicenter of different spinal columns. FA, MD, and $\lambda_{\perp}$ altered more than two SD at the injury epicenter of right lateral, dorsal, and ventral columns. $\lambda_{\|}$altered more than two SD at the injury epicenter of ventral column. MTCSF changed more than two SD at the injury epicenter of the left lateral, dorsal, and ventral column, below the injury epicenter at the right lateral, dorsal, and ventral column, and above the injury epicenter at the dorsal column. All DTIderived quantities values for the left lateral column were within the normal range, suggesting the left lateral column is the most well preserved spinal column. This corresponds with the patient's 
Table 3 | Injury region specific data comparison of controls.

\begin{tabular}{|c|c|c|c|c|}
\hline & \multicolumn{4}{|c|}{$\begin{array}{c}\text { Controls } \\
\text { Mean } \pm \text { SD }\end{array}$} \\
\hline & Below & Epicenter & Above & Total \\
\hline \multicolumn{5}{|l|}{ Left lateral } \\
\hline FA & $0.68 \pm 0.10$ & $0.74 \pm 0.10$ & $0.79 \pm 0.09$ & $0.75 \pm 0.10$ \\
\hline $\mathrm{MD}\left(\mu \mathrm{m}^{2} / \mathrm{ms}\right)$ & $0.99 \pm 0.16$ & $1.00 \pm 0.24$ & $0.93 \pm 0.25$ & $0.97 \pm 0.22$ \\
\hline$\lambda_{\|}\left(\mu \mathrm{m}^{2} / \mathrm{ms}\right)$ & $1.89 \pm 0.23$ & $2.01 \pm 0.34$ & $1.98 \pm 0.34$ & $1.98 \pm 0.29$ \\
\hline$\lambda_{\perp}\left(\mu \mathrm{m}^{2} / \mathrm{ms}\right)$ & $0.54 \pm 0.18$ & $0.51 \pm 0.23$ & $0.42 \pm 0.21$ & $0.47 \pm 0.22$ \\
\hline MTCSF & $0.48 \pm 0.08$ & $0.48 \pm 0.11$ & $0.48 \pm 0.09$ & $0.49 \pm 0.10$ \\
\hline \multicolumn{5}{|l|}{ Right lateral } \\
\hline FA & $0.74 \pm 0.09$ & $0.79 \pm 0.07$ & $0.78 \pm 0.09$ & $0.78 \pm 0.08$ \\
\hline $\mathrm{MD}\left(\mu \mathrm{m}^{2} / \mathrm{ms}\right)$ & $0.98 \pm 0.18$ & $0.89 \pm 0.16$ & $0.94 \pm 0.23$ & $0.94 \pm 0.21$ \\
\hline$\lambda_{\|}\left(\mu \mathrm{m}^{2} / \mathrm{ms}\right)$ & $1.99 \pm 0.22$ & $1.90 \pm 0.26$ & $1.97 \pm 0.32$ & $1.98 \pm 0.30$ \\
\hline$\lambda_{\perp}\left(\mu \mathrm{m}^{2} / \mathrm{ms}\right)$ & $0.48 \pm 0.19$ & $0.39 \pm 0.13$ & $0.43 \pm 0.21$ & $0.43 \pm 0.19$ \\
\hline MTCSF & $0.48 \pm 0.10$ & $0.48 \pm 0.10$ & $0.48 \pm 0.08$ & $0.49 \pm 0.10$ \\
\hline \multicolumn{5}{|l|}{ Dorsal } \\
\hline FA & $0.70 \pm 0.14$ & $0.78 \pm 0.09$ & $0.77 \pm 0.08$ & $0.76 \pm 0.10$ \\
\hline $\mathrm{MD}\left(\mu \mathrm{m}^{2} / \mathrm{ms}\right)$ & $1.11 \pm 0.31$ & $0.98 \pm 0.17$ & $1.02 \pm 0.15$ & $1.04 \pm 0.22$ \\
\hline$\lambda_{\|}\left(\mu \mathrm{m}^{2} / \mathrm{ms}\right)$ & $2.14 \pm 0.36$ & $2.10 \pm 0.22$ & $2.17 \pm 0.20$ & $2.16 \pm 0.28$ \\
\hline$\lambda_{\perp}\left(\mu \mathrm{m}^{2} / \mathrm{ms}\right)$ & $0.59 \pm 0.32$ & $0.42 \pm 0.17$ & $0.45 \pm 0.16$ & $0.49 \pm 0.22$ \\
\hline MTCSF & $0.47 \pm 0.08$ & $0.48 \pm 0.10$ & $0.46 \pm 0.09$ & $0.47 \pm 0.09$ \\
\hline \multicolumn{5}{|l|}{ Ventral } \\
\hline FA & $0.59 \pm 0.07$ & $0.61 \pm 0.07$ & $0.66 \pm 0.08$ & $0.61 \pm 0.08$ \\
\hline $\mathrm{MD}\left(\mu \mathrm{m}^{2} / \mathrm{ms}\right)$ & $1.10 \pm 0.17$ & $1.15 \pm 0.25$ & $1.09 \pm 0.20$ & $1.16 \pm 0.25$ \\
\hline$\lambda_{\|}\left(\mu \mathrm{m}^{2} / \mathrm{ms}\right)$ & $1.92 \pm 0.28$ & $2.03 \pm 0.36$ & $2.03 \pm 0.29$ & $2.05 \pm 0.35$ \\
\hline$\lambda_{\perp}\left(\mu \mathrm{m}^{2} / \mathrm{ms}\right)$ & $0.69 \pm 0.14$ & $0.71 \pm 0.22$ & $0.62 \pm 0.18$ & $0.71 \pm 0.22$ \\
\hline MTCSF & $0.52 \pm 0.11$ & $0.52 \pm 0.08$ & $0.51 \pm 0.09$ & $0.54 \pm 0.11$ \\
\hline
\end{tabular}

Imaging parameters (FA, MD, $\lambda_{\|}, \lambda_{\perp}$, and MTCSF) were measured for cervical spinal cord regions that correspond with the subject's above the injury, injury epicenter, and below the injury region of the subject. Values reported are mean $\pm S D$ of each injury region.

clinical presentation, who presents higher motor score for the left side of the body than the right.

\section{BRAIN DTI DATA ANALYSIS}

The structural integrity of the patient's brain was investigated through T1-w imaging and DTI, using an atlas-based analysis (ABA) method (Faria et al., 2010, 2011). The diffusion weighted images were first co-registered to one of the b0 images using a 12-mode affine transformation of Automated Image Registration (AIR) (Woods et al., 1998). The six elements of the diffusion tensor, FA, and mean diffusivity (MD) were calculated. After skull-stripping, the images were first normalized to the JHU-DTIMNI "Eve" template (Jiang et al., 2006) with a nine-parameter affine transformation of AIR using b0 image. Then, a non-linear transformation using dual-contrast large deformation diffeomorphic metric mapping (LDDMM) (Beg et al., 2005) was applied, using FA and MD images. These procedures are reciprocal, so the inverse-transformed brain parcellation map was superimposed onto the original MRI images, and led to parcellation of the brain into 130 anatomical structures (Oishi et al., 2008, 2011). Some of the structures were then further segmented into gray and white matter using an FA threshold of 0.25 in each subject, followed by minimum manual adjustment. This resulted in 46 additional parcellations, yielding a total of 176 regions. When the quantitative values were obtained, the cerebrospinal fluid spaces were excluded by an MD threshold at $0.0020 \mathrm{~mm}^{2} / \mathrm{s}$. The final results of the anatomical quantification included volume, FA and MD for each subject. The non-linear image transformation and the atlas-based parcellation were performed using DiffeoMap and RoiEditor (http://www.MriStudio.org) (Oishi et al., 2008).

\section{BRAIN RESTING STATE FMRI PREPROCESSING AND ANALYSIS}

The preprocessing of rs-fMRI data was performed using SPM8 (www.fil.ion.ucl.ac.uk/spm) (Friston et al., 1994) and Matlab (Natick, MA). The preprocessing pipeline involved: (1) slice timing correction, (2) motion correction, (3) co-registration, (4) unified segmentation-normalization (Ashburner and Friston, 2005), (5) high pass filtering with $0.005 \mathrm{~Hz}$ cutoff, and (6) a spatial smoothing using $6 \mathrm{~mm}$ full-width at half-maximum Gaussian kernel. Finally, principal component analysis was performed, followed by group independent component analysis (GICA) (Calhoun et al., 2001) of all runs from the healthy controls and the subject using the GIFT software (http://mialab.mrn. org/software/gift). An order selection using minimum description length (MDL) criterion (Li et al., 2007) was performed and a total of 39 independent components were initially estimated. After GICA, three were eliminated due to low similarity measures obtained using ICASSO toolbox; (Himberg et al., 2004) 24 were rejected due to artifacts (e.g., motion) and physiology (e.g., cardiac pulsation).

For each remaining IC, the magnitude of temporal fluctuations was computed to provide a measure of within-network connectivity (WNC). This was done by first converting the back reconstructed time courses to percent signal change measurements for normalization purpose, and computing the root mean square (RMS) of the normalized time course values. The between network connectivity (BNC) was computed for every pair of functional networks as well, as the Pearson's correlation of the network time courses.

\section{RESULTS}

\section{MRI-BASED STRUCTURAL EVALUATION OF A CHRONICALLY INJURED SPINAL CORD}

In the T2-weighted MR images, a lesion is visible in C4-C5 of the injured spinal cord (Figures 2A,B), location of which agrees with the known location of the injury epicenter. No lesion is observed in a healthy spinal cord (Figures 2E,F).

The degree of preservation of longitudinally oriented axonal tracts in white matter was investigated by assessing the whole cord and white matter atrophy of the patient's spinal cord. We measured wca (Figure 3A) and wma (Figure 3B) of each slice in the cervical cord for the controls (mean \pm SD; SD: standard deviation; blue line) and the patient (mean; red line) using FA maps (Figures 2D,H). Gray boxes in each profile represent the injury epicenter. The wca and wma are reduced throughout the cervical cord (more than one SD decrease) and maximally reduced in the 


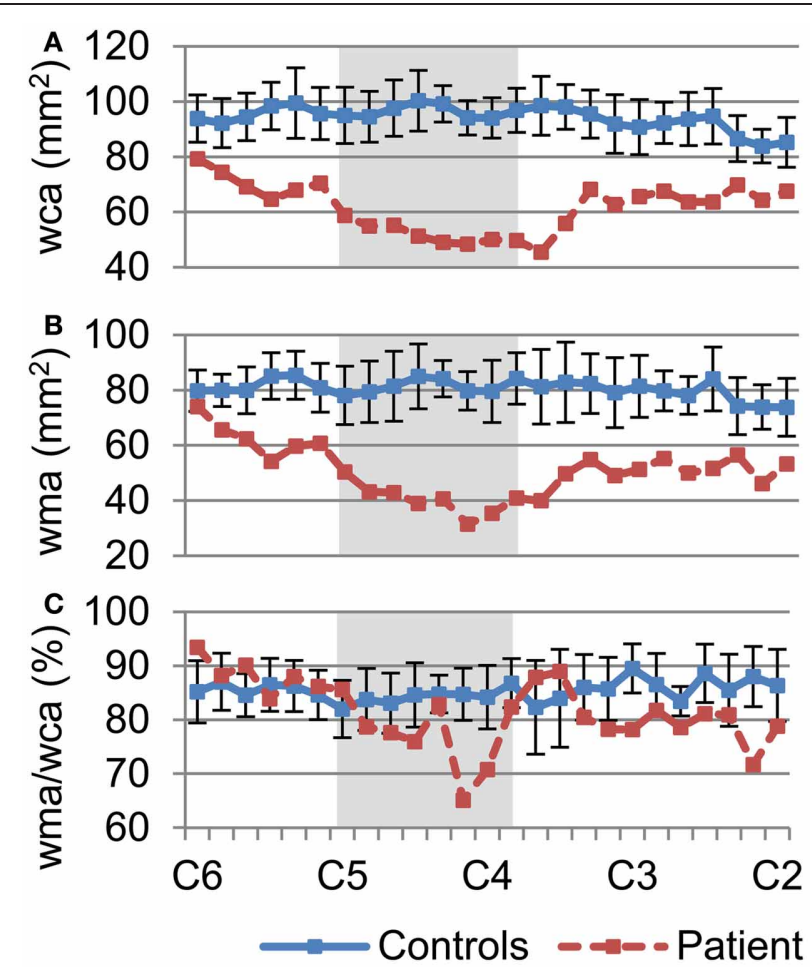

FIGURE 3 | Quantification of whole cord and white matter atrophy, as a measure of white matter tissue sparing throughout the cervical spinal cord. (A) Degree of whole cord atrophy, as measured by the whole cord area, (B) white matter area, and $\mathbf{( C )}$ the ratio of wma over wca, was measured between C2-C6. Values of the controls (blue line) are mean \pm SD. Plot of wca $(\mathbf{A})$ and wma $(\mathbf{B})$ demonstrate atrophy of the patient's spinal cord not only at the injury epicenter (dark area) but throughout the cervical cord. Plot of ratio of wma over wca (C) shows marked decrease of white matter around the injury epicenter.

injury epicenter (55 and 62\%, respectively). These profiles show that atrophy spans the entire measured area within the cervical cord but is more pronounced in the injury epicenter. In addition, a measure of the area of spared white matter tissue was provided by the wma to wca ratio (Figure 3C). A sharp decrease of the ratio (23\%) shows that the focal atrophy at the epicenter of the injury is due to white matter atrophy.

We aimed to assess the axonal integrity and myelination within major spinal cord columns (dorsal, lateral, and ventral columns) of the spared white matter tissue, where dorsal columns convey sensory function while the lateral and ventral columns convey primarily motor functions. Segmentation of each of the columns was performed using DTI fiber tractography, and column profiles for each of the DTI- and MTI-derived quantities were created (Figure 4). Quantities derived from DTI [FA, MD, axial diffusivity $\left(\lambda_{\|}\right)$, and radial diffusivity $\left(\lambda_{\perp}\right)$ ] and MTI (MTCSF) have been previously shown to be sensitive to axonal and myelin integrity (Beaulieu, 2002; Song et al., 2002, 2005; Smith et al., 2005; Farrell et al., 2010; Landman et al., 2010; Levesque et al., 2010; Choe et al., 2012), with DTI-derived quantities more sensitive to axon and MTI-derived quantities more sensitive to myelin. Next, we used MTCSF images to place ROIs for DTI tractography. MTCSF increased by more than one SD at the injury epicenter in all spinal columns, and its changes were diffuse and extended throughout the cervical cord. Unlike MTCSF, FA decreased by more than one SD primarily at the injury epicenter along all spinal columns, with the greatest decrease observed in the dorsal column. The decrease in FA was more pronounced in the right lateral column than in the left lateral column (Figure 4). These observations are consistent with the patient's neurological manifestation, as the patient's sensory deficit is worse than motor deficit and motor deficit on the right side of the body is worse than left side of the body. MD, $\lambda_{\|}$, and $\lambda_{\perp}$ increased focally at the injury epicenter. However, the pattern of changes of these quantities is different from that of FA, with changes being more pronounced in the right versus left lateral column and the ventral column being the most pronounced (Figure 4, Tables 2, 3).

\section{MRI-BASED EVALUATION OF THE BRAIN IN A CHRONIC SPINAL CORD INJURY PATIENT \\ Structural imaging}

Considering the extensive structural changes we observed in the spinal cord of the patient, we wanted to assess if such changes extend to the brain. The structural integrity of the patient's brain was investigated through T1-w imaging and DTI, using an atlas based whole brain analysis with 176 parcellations. Structure volume, FA, and MD measurements of appropriate controls and the patient were compared using a Mann-Whitney U-test. No significant differences between the controls and the patient were observed (Table 4). The lack of alterations of these quantities indicates the SCI has not produced significant structural changes in the patient's brain.

\section{Resting state fMRI (rs-fMRI)}

In order to investigate cortical plasticity, we used rs-fMRI, which measures spontaneous fluctuations in BOLD (blood oxygenation level dependent) signal in the absence of externally-cued task. This allows for direct comparison with controls regardless of a patient's motor and sensory deficits. Functional networks were identified using GICA, which uses higher-order statistics to recover independent sources from multivariate data (Calhoun et al., 2001). It separates rs-fMRI data into components reporting on spontaneous fluctuations in intrinsic brain networks, and components reporting on nuisances such as head motion and cardiac pulsations. The procedure yielded 12 independent components (IC) representing different functional networks (Figure 5).

Within-network connectivity: For each IC, the magnitude of temporal fluctuations - the root mean square (RMS) of the component time course-provides a measure of within-network connectivity. The RMS \% BOLD changes for each IC of appropriate controls and the patient are shown as series of box plots in Figure 5. The outliers in the plot were determined using a variable $w$ (maximum whisker length), which was set to 1.5. Points were drawn as outliers if they are larger than $\mathrm{q} 3+w(\mathrm{q} 3-\mathrm{q} 1)$ or smaller than $\mathrm{q} 1-w(\mathrm{q} 3-\mathrm{q} 1)$, where $\mathrm{q} 1$ and $\mathrm{q} 3$ are the 25 th and 75 th percentiles, respectively. The plotted whisker extends to the adjacent value, which is the most extreme data value that is not an outlier. The median temporal modulations of the controls' ICs are 


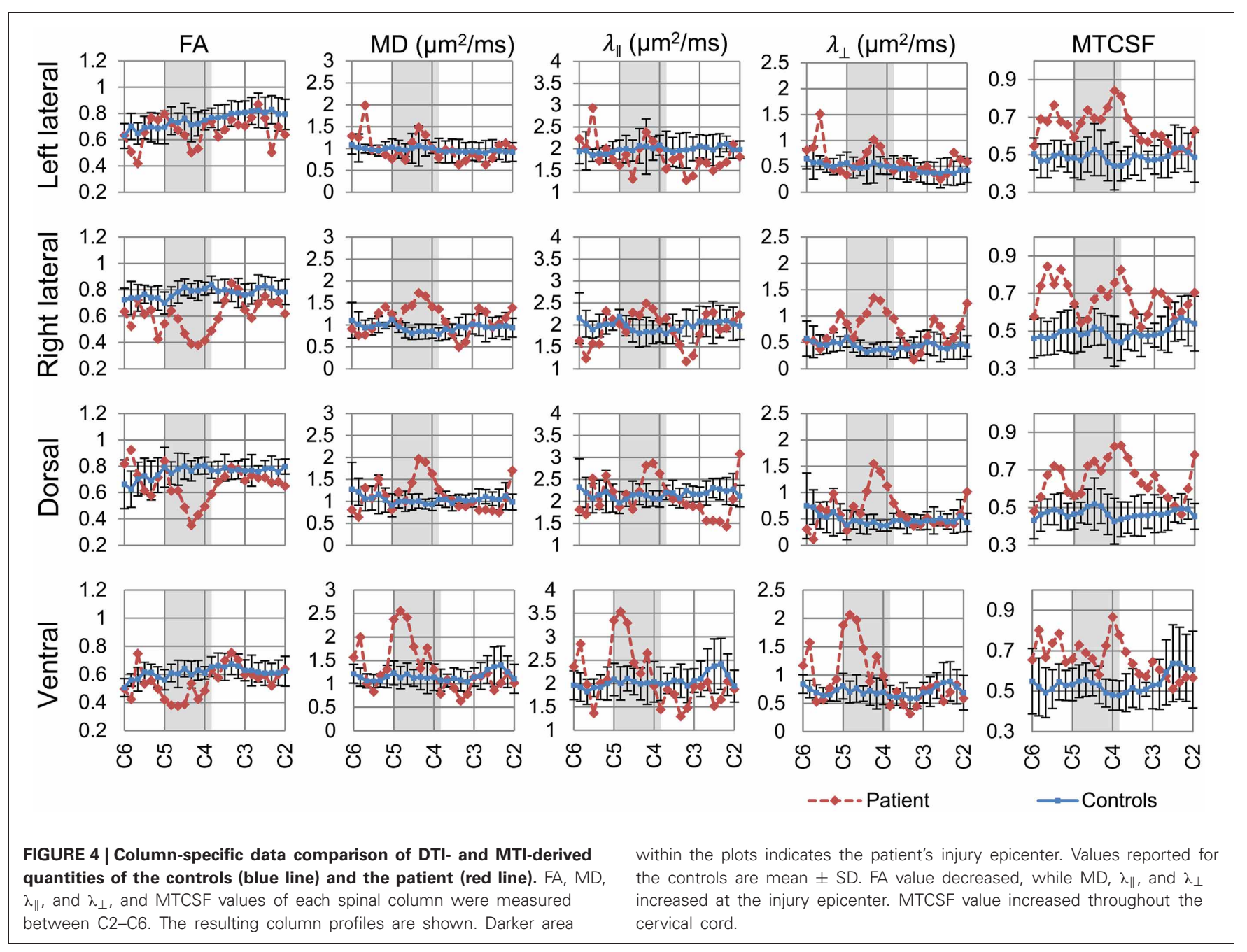

between 0.3 and $0.5 \%$ of original BOLD MRI signal. The median temporal modulations of the patient's ICs are between 0.4 and $1.4 \%$ of average BOLD MRI signal, except for the dorsal attention and left executive networks with higher temporal modulation at 1.6 and $1.9 \%$, respectively. The range of temporal modulations of the patient's ICs were bigger for auditory, dorsal somatosensory, secondary visual, and dorsal attention networks, compared to those of the controls. In general, magnitude of the network temporal modulation of the patient was bigger than that of the controls.

Between-network connectivity: Synchrony between network time courses is referred to as between-network connectivity (BNC) (Joel et al., 2011). Figures 6A,B show a combined correlation matrix of the controls and the patient, and a combined matrix of the standard deviation of the corresponding BNC measurements, respectively. Figure 6C shows the difference between the controls and the patient's BNC measurements. Figure 6D shows box plots of the controls' BNC measurements, with the mean BNC value of corresponding functional networks of the patient indicated using red dots. Ten functional networks with the biggest mean $\mathrm{BNC}$ value difference between the controls and the patient are shown. Compared to controls, five functional networks of the patient, namely the sensorimotor, visual, DMN, attention, and executive networks, show increased synchrony with other networks. A decrease of functional connectivity is also observed between the left and right executive networks. The visual networks show a marked increase of connectivity with other functional networks such as the DMN, with the largest increase in the connectivity being observed between sensorimotor and visual networks. The patient has not regained sensory function in the lower body and relies heavily on vision to guide his lower body motor tasks. The increased connectivity between the sensorimotor and the visual networks is consistent with this clinical presentation and suggests compensatory functional changes in the patient's brain.

\section{DISCUSSION}

We used advanced MRI technologies to understand how a patient with a complete SCI achieved a pragmatic cure, defined as sufficient recovery of motor, sensory, and autonomic function to enable the patient to live and function independently. The patient achieved the recovery despite a significant atrophy, a focal FA decrease, and a diffuse increase of MTCSF in the residual white matter. This indicates that the patient's residual spinal cord tissue 


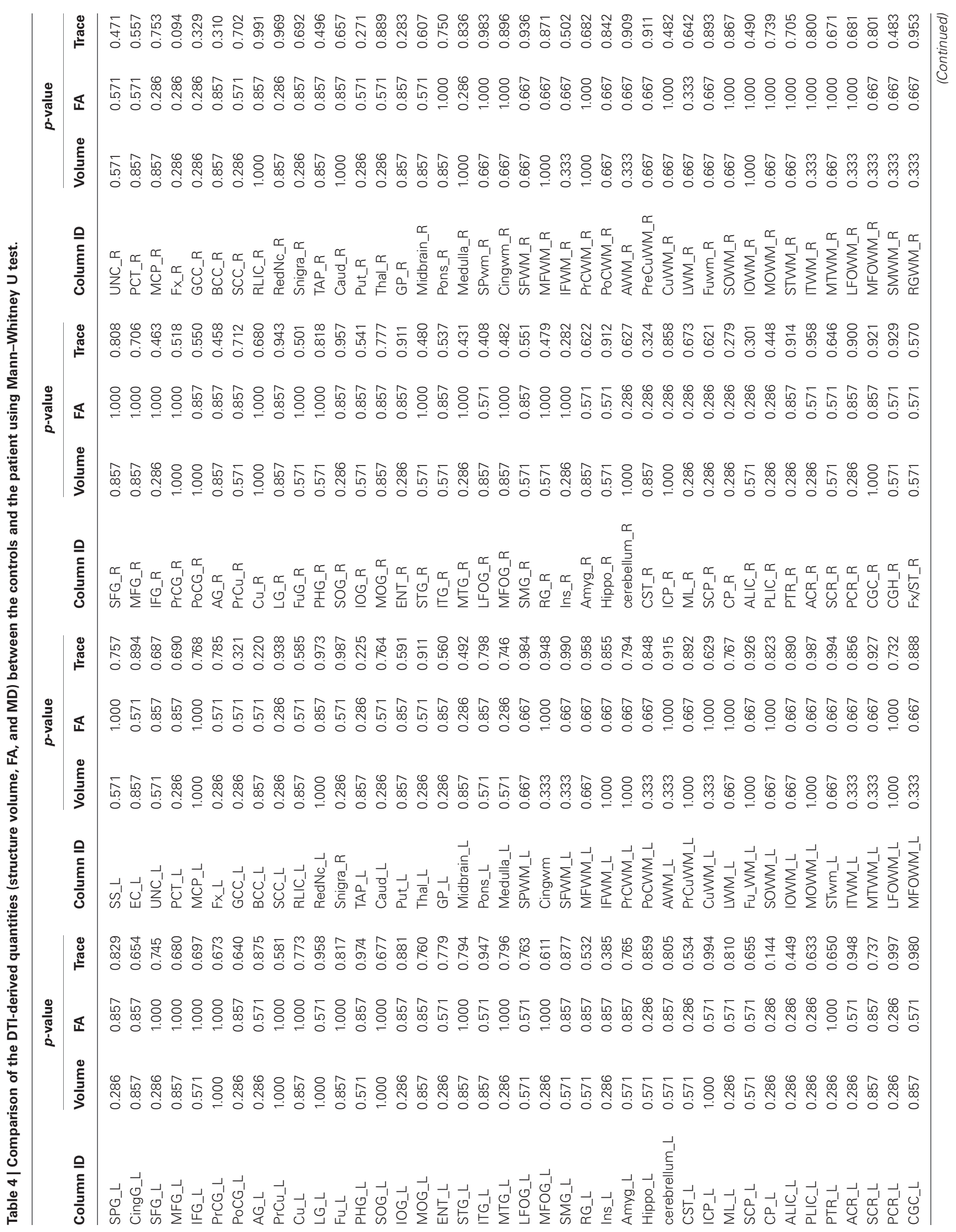




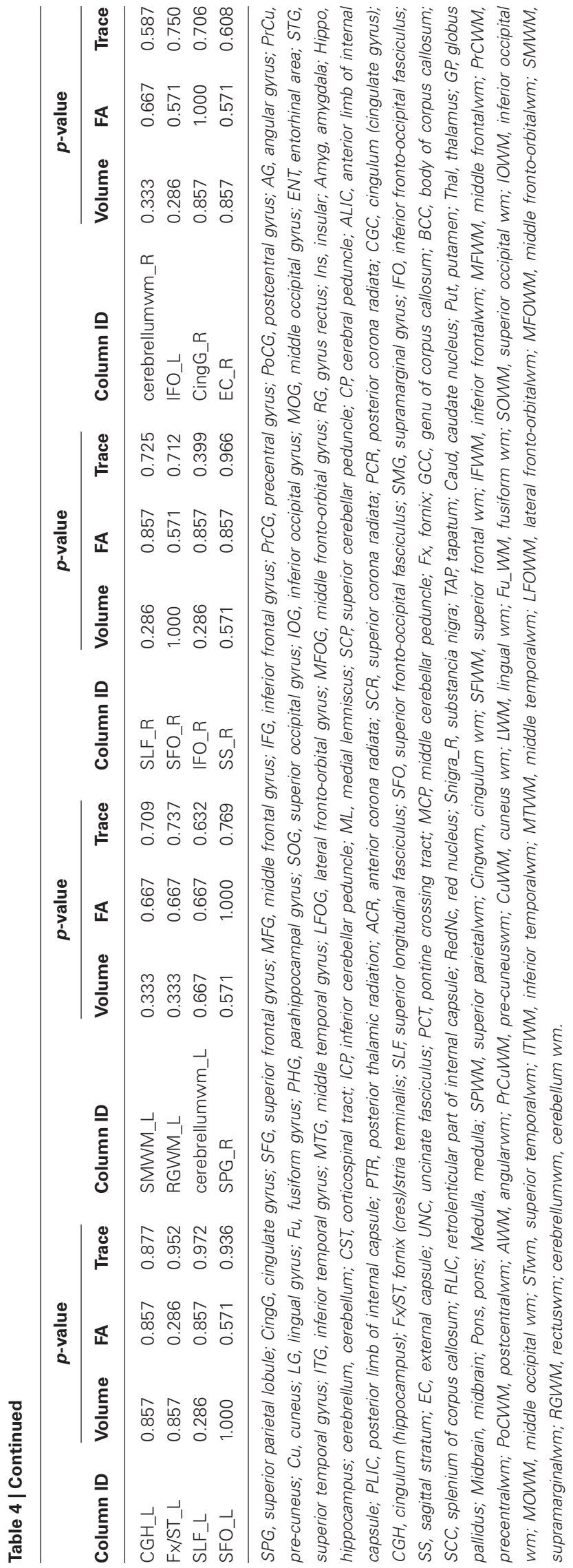

has sufficient integrity to enable function. The rs-fMRI showed increased functional connectivity between the patient's sensorimotor and the visual networks, indicating cortical plasticity; this cortical plasticity was not accompanied by structural changes in the brain.

\section{MRI-BASED STRUCTURAL EVALUATION OF A CHRONICALLY INJURED SPINAL CORD}

Whole cord cross-sectional area was shown to correlate with the motor and sensory scores of SCI patients (Lundell et al., 2011). However, the atrophy of the whole cord is accompanied by increased scar tissues in the cord and the degree of white matter atrophy can be greater than that of the whole cord, as our data shows (Figure 3). We used FA images, which can differentiate white matter from gray matter and scar tissues, to segment white matter from the injured cord. The wca and wma values of the healthy spinal cords measured using this approach were comparable to those values measured using higher resolution structural images acquired through 7T MRI (Sigmund et al., 2011), CT (Fountas et al., 1998), cadaver anatomy (Ko et al., 2004), as well as other previous histological methods (Kameyama et al., 1996; White et al., 1997; Gilmore et al., 2005). Our results showed that the patient's neurological recovery is achieved despite a $62 \%$ atrophy of the white mater tissue at the injury epicenter.

Structural integrity of the major columns directly affects neurological recovery of specific motor and sensory functions. Column-specific data analysis (Smith et al., 2010) enabled incorporation of manual and tractography-based ROI selection approach, allowing more time efficient data analysis, and comparison of DTI- and MTI-derived quantities of the controls and the patient in the residual tissue from the major columns (Figure 4). DTI and MTI are advanced MR imaging modalities that are sensitive to tissue microstructures. DTI provides an indirect measure of tissue structure on a microscopic scale by probing water diffusion (Basser et al., 1994), while MTI does so by measuring the magnetization interaction between bulk water protons and semi-solid macromolecular protons (Wolff and Balaban, 1989; Smith et al., 2005). FA, $\lambda_{\|}$, and MTCSF are sensitive to changes in both axonal and myelin integrity, but previous studies have shown that $\lambda_{\|}$is more sensitive to axonal damages (Song et al., 2002, 2005; Budde et al., 2009) while FA is not specific (Farrell et al., 2010; Landman et al., 2010). In our patient, focal changes of DTI-derived quantities (Figure 4) were found that indicate mixed damage types over the cervical cord-i.e., the reduction in FA is caused mainly by increases in $\lambda_{\|}$that could be due to axonal and myelin damage. MTCSF is more sensitive to myelination and the diffuse increase of MTCSF throughout the cervical cord (Figure 4) suggests diffuse demyelination or dysmyelination throughout the cervical cord. Overall, the DTI parameters describe the extensive damage but not how it affected the recovery.

A high correlation between FA and total AIS scores in chronic SCI patients has been described (Cohen-Adad et al., 2011). From that data we extrapolate a FA value (0.47) for chronic SCI patients with the same AIS score as our patient (124). The FA value of the patient $(0.63)$ is higher than the extrapolated FA value $(0.47)$. The 


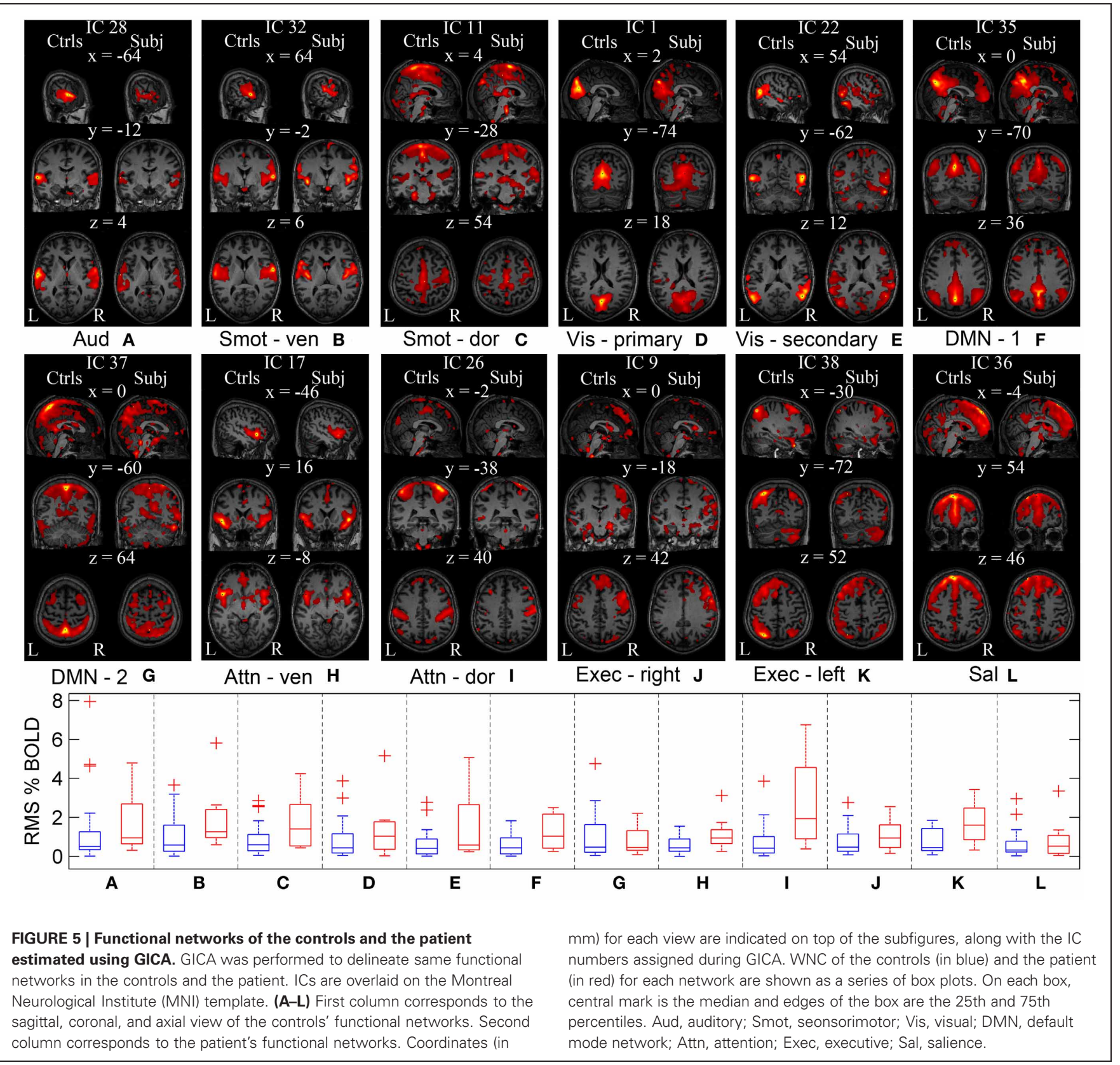

higher FA of the patient is driven by the higher $\lambda_{\|}$. The higher $\lambda_{\|}$ value can be explained in part by the previously reported trend of $\mathrm{MD}$ and $\lambda_{\|}$change in different stages of traumatic injuries, with initial decrease during the acute phase of injury (Loy et al., 2007) and increased values during the chronic phase of injury (Naismith et al., 2009; Klawiter et al., 2011). This trend alone, however, does not fully explain the higher $\lambda_{\|}$observed in the patient, as the patient cohort observed in Cohen-Adad et al. (2011) are also chronic SCI patients with comparable delay after injury as the patient in this report. The higher $\lambda_{\|}$of the patient therefore may suggest higher axonal integrity. The DTI- and MTI-derived quantities of the controls were comparable to those reported in similar studies (Zackowski et al., 2009; Smith et al., 2010).
The small size of a spinal cord presents major imaging challenges. High resolution images are often desired, and the size makes the images more vulnerable to partial volume effect and various motions artifacts. While longer scan time and gating may be used to alleviate these issues to an extent, keeping patients with movement difficulties in the scanner for a long time is undesirable. Issue of misregistration cannot be ignored either, as the consequence of misregistration is greater in the spinal cord due to its size. One source of such misregistration is the use of an EPI read out during DTI acquisition, which can result in substantial eddy current effects. In order to minimize the effect of eddy current and misregistration, a robust registration scheme utilizing CATNAP (Farrell et al., 2007; Landman et al., 2007) was 
A Controls (bottom left) / Patient (top right)

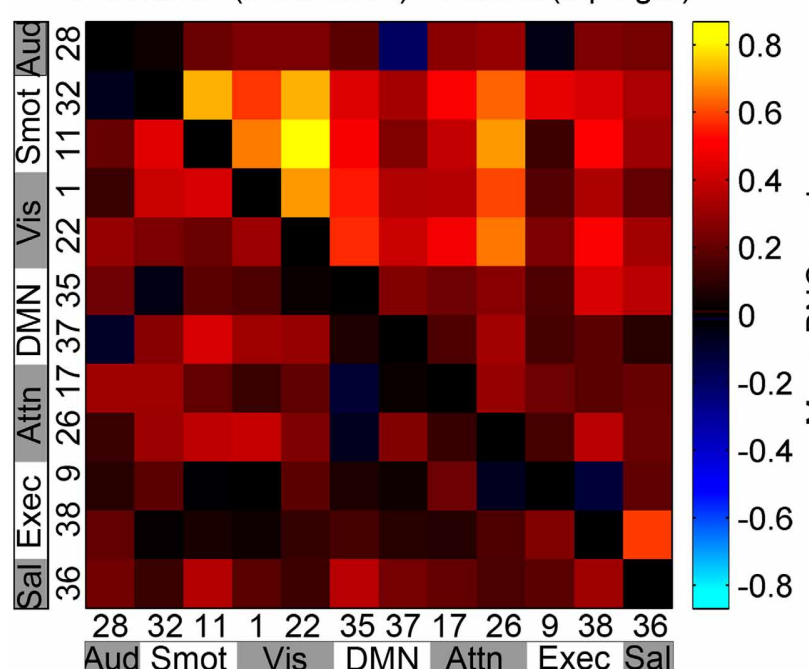

c BNC value difference

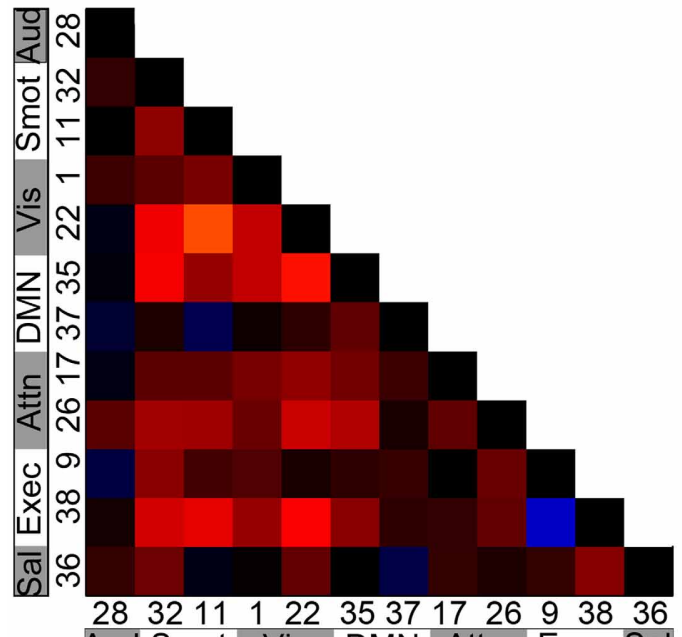

Aud Smot Vis DMN Attn Exec Sal

FIGURE 6 | Between-network connectivity. (A) BNC correlation matrix shows synchrony between pairs of functional networks. (B) Combined matrix of the standard deviation of the corresponding BNC measurements. $(\mathbf{A}, \mathbf{B})$ In reference to the top-left to bottom-right diagonal axis, bottom-left portion corresponds to the controls and top-right portion corresponds to the patient.
B Controls (bottom left) / Patient (top right)

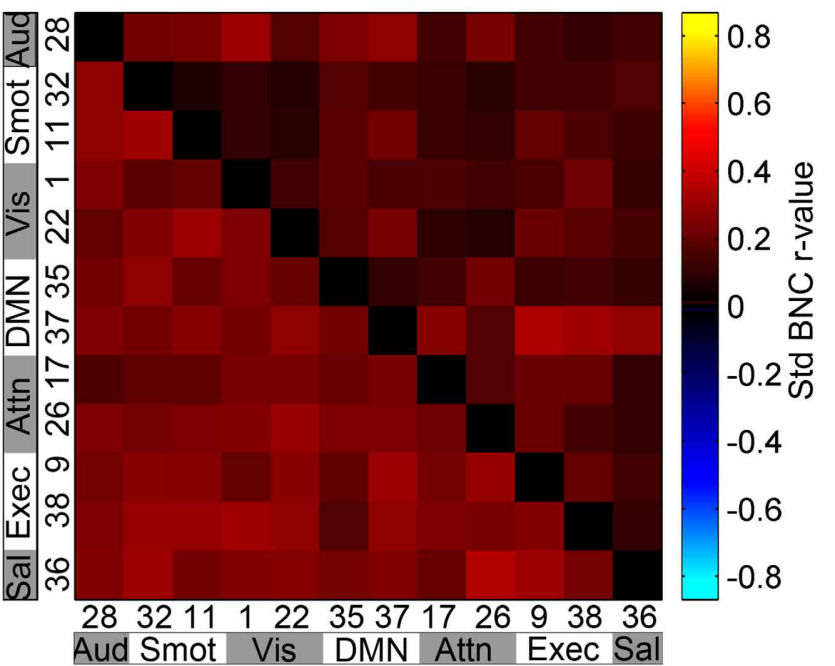

D BNC r-value of the patient (mean; red dots) and the controls (box plots)

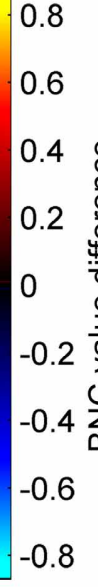

Diagonal elements have been zeroed for display purposes. (C) Difference between the controls and the patient's BNC measurements. (D) Box plots of controls' BNC measurements, with the patient's mean BNC values indicated by red dots. Shown are the 10 functional networks with the largest differences between the patient and controls.

used to correct artifacts in DTI, using optimized input parameters (Farrell et al., 2008). While a more sophisticated eddy current correction scheme that uses field maps may improve the registration accuracy further, the tract-specific data comparison of this study showed good correlation between structural and functional information provided by MRI and clinical evaluation of the patient.

\section{MRI-BASED EVALUATION OF THE BRAIN IN A CHRONIC SPINAL CORD INJURY PATIENT}

Considering the extensive structural changes of the patient's spinal cord, and based on previous studies that report marked atrophy in the sensorimotor cortex (Crawley et al., 2004; Jurkiewicz et al., 2006; Wrigley et al., 2009; Freund et al., 2011; Henderson et al., 2011), our initial hypothesis was that we would observe similar gray matter atrophy in the patient's brain. Our result indicates, however, that the injury did not lead to significant structural changes in the patient's brain. One possible explanation is that unlike other SCI patients, the patient's structural changes were small and intracortical, and we did not have enough sample size and statistical power to detect the subtle changes. One study (Henderson et al., 2011), for example, shows that an extensive functional reorganization such as the one the patient demonstrates, may work to minimize the anatomical atrophy in the 
sensorimotor cortex. This hypothesis is also in part supported by the initial observations of chronic deafferentiation and its effect on cortical anatomy (Florence et al., 1998) where major functional reorganization following limb amputation of a macaque monkey was accompanied by significant increase of intracoritcal connections but not by any significant changes of major thalamocortical fiber tracts.

In order to differentiate functional networks, GICA (Calhoun et al., 2001) was performed using all eight runs of the subject and one run from 20 healthy controls. This enabled us to identify similar functional networks in both the control group and the subject, effectively eliminating the necessity to perform ICA separately on each subject and allowing differentiation of the same functional networks with different activation patterns. While there have been some concerns that the identification of aggregate functional networks using a single dataset of multiple groups may bias the results, a simulation study (Schmithorst and Holland, 2004) showed that GICA can identify a functional network that is present in only $10-15 \%$ of the total population. Another advantage of GICA is that the method is model-free and does not make any assumptions about the nature of BNC.

A novel finding of this study is the increased connectivity between sensorimotor and visual networks. The patient regained only $10 \%$ of sensory function in the lower body and relies heavily on vision to guide his movements. In light of this clinical presentation, our observation suggests plastic changes in the sensorimotor and visual networks of the patient. Studies on stroke patients have shown that visual guidance of movement contributes to the recovery of hand motor function (Kalra et al., 1997; Seitz et al., 1999), while lack of it may be detrimental for the recovery of sensorimotor function (Kalra et al., 1997). The increased functional connectivity between the sensorimotor and visual networks suggests that deprivation of sensory input that resulted from the SCI may have led to compensatory functional changes in the patient's brain. Another possibility is that the patient had increased connectivity between the two networks prior to the injury and this contributed to his recovery.

We also observed increased connectivity of the left executive network to other brain networks. The executive network (Seeley et al., 2007) links dorsolateral frontal and parietal cortex and is a task positive network that is related to working memory and control processes (Dosenbach et al., 2007; Stevens, 2009). The asymmetrical nature of the increased connectivity (left more than

\section{REFERENCES}

Ashburner, J., and Friston, K. J. (2005). Unified segmentation. Neuroimage 26, 839-851. doi: 10.1016/j.neuroimage.2005.02.018

Basser, P. J., Mattiello, J., and LeBihan, D. (1994). MR diffusion tensor spectroscopy and imaging. Biophys. J. 66, 259-267. doi: 10.1016/S00063495(94)80775-1

Beaulieu, C. (2002). The basis of anisotropic water diffusion in the nervous system - a technical review. NMR Biomed. 15, 435-455. doi: 10.1002/nbm.782
Beg, M., Miller, M., Trouve, A., and Younes, L. (2005). Computing large deformation metric mappings via goodesic flows of diffeomorphisms. Int. J. Comput. Vis. 61, 139-157. doi: $10.1023 /$ B:VISI.0000043755. 93987.aa

Belegu, V., Oudega, M., Gary, D. S., and McDonald, J. W. (2007). Restoring function after spinal cord injury: promoting spontaneous regeneration with stem cells and activitybased therapies. Neurosurg. Clin. North Am. 18, 143-168, xi. doi: 10.1016/j.nec.2006.10.012

right) may be related to the asymmetric recovery of motor and sensory function of the patient. It should be noted that the magnitude of the asymmetry of the motor and sensory function is not fully appreciated through the AIS score, which is well-suited to describe the neurological level of the injury but ill-suited to describe the degree of functional loss.

The study presented here is a case report; therefore we are limited in our ability to draw statistically significant conclusions-for spatial differences of ICs as well as changes in BNC. This is because we are comparing single run data from multiple controls, subject to an inter-subject variability, to multiple run data from the subject, subject to an intra-subject variability. A larger cohort of SCI population is necessary in order to draw statistically significant conclusion about the changes in ICs and BNC of SCI patients. Nevertheless, the study showed good agreement between the rs-fMRI results and the clinical presentation of the subject and presents the possibility that rs-fMRI may aid in better SCI diagnosis and prognosis, as well as better monitoring and optimizing therapies for SCI subjects.

\section{CONCLUDING REMARKS}

In conclusion, the current study provides a detailed analysis of structural and functional changes in the central nervous system of a patient with chronic SCI who underwent a remarkable neurological recovery that continued for 17 years. To the best of our knowledge, this is the first study which used rs-fMRI to investigate cortical functional changes in SCI patients, alongside the investigation of structural changes using spinal cord imaging. Furthermore, the study has theoretical significance because it may identify variables-i.e., sufficient structural integrity for proper signal conduction in the spinal cord and compensatory plastic changes in the brain-that are necessary for neurological and functional improvements. Additionally, this work shows the potential of rs-fMRI to evaluate the extent of cortical plasticity required for functional recovery in patients whose neurological deficits prohibit completion of externally cued motor or sensory tasks.

\section{ACKNOWLEDGMENTS}

The authors would like to thank Dr. Jeffrey D. Rothstein for helpful discussion and advice. This work was supported in part by grants from the DOD (W81XWH-08-1-0192) and NIH (R21 EB006120-01A2 and P41 EB015909).

Blight, A. R. (1983). Cellular morphology of chronic spinal cord injury in the cat: analysis of myelinated axons by line-sampling. Neuroscience 10, 521-543. doi: 10.1016/0306-4522(83)90150-1

Budde, M. D., Xie, M., Cross, A. H., and Song, S. K. (2009). Axial diffusivity is the primary correlate of axonal injury in the experimental autoimmune encephalomyelitis spinal cord: a quantitative pixelwise analysis. J. Neurosci. 29, 2805-2813. doi: 10.1523/JNEUROSCI.460508.2009
Calhoun, V. D., Adali, T., Pearlson, G. D., and Pekar, J. J. (2001). A method for making group inferences from functional MRI data using independent component analysis. Hum. Brain Mapp. 14, 140-151. doi: 10.1002/hbm. 1048

Choe, A. S., Stepniewska, I., Colvin, D. C., Ding, Z., and Anderson, A. W. (2012). Validation of diffusion tensor MRI in the central nervous system using light microscopy: quantitative comparison of fiber properties. NMR Biomed. 25, 900-908. doi: 10.1002/nbm.1810 
Cohen-Adad, J., El Mendili, M. M., Lehericy, S., Pradat, P. F., Blancho, S., Rossignol, S., et al. (2011). Demyelination and degeneration in the injured human spinal cord detected with diffusion and magnetization transfer MRI. Neuroimage 55, 1024-1033. doi: 10.1016/j.neuroimage.2010.11.089

Cohen-Adad, J., El Mendili, M. M., Morizot-Koutlidis, R., Lehericy, S., Meininger, V., Blancho, S., et al. (2013). Involvement of spinal sensory pathway in ALS and specificity of cord atrophy to lower motor neuron degeneration. Amyotroph. Lateral Scler. Frontotemporal Degener. 14, 30-38. doi: 10.3109/17482968.2012.701308

Crawley, A. P., Jurkiewicz, M. T., Yim, A., Heyn, S., Verrier, M. C., Fehlings, M. G., et al. (2004). Absence of localized grey matter volume changes in the motor cortex following spinal cord injury. Brain Res. 1028, 19-25. doi: 10.1016/j.brainres.2004.08.060

Dosenbach, N. U., Fair, D. A., Miezin, F. M., Cohen, A. L., Wenger, K. K., Dosenbach, R. A., et al. (2007). Distinct brain networks for adaptive and stable task control in humans. Proc. Natl. Acad. Sci. U.S.A. 104, 11073-11078. doi: 10.1073/pnas.0704320104

Endo, T., Spenger, C., Tominaga, T., Brene, S., and Olson, L. (2007). Cortical sensory map rearrangement after spinal cord injury: FMRI responses linked to nogo signalling. Brain 130, 2951-2961. doi: 10.1093/ brain/awm 237

Faria, A. V., Hoon, A., Stashinko, E., Li, X., Jiang, H., Mashayekh, A., et al. (2011). Quantitative analysis of brain pathology based on MRI and brain atlasesapplications for cerebral palsy. Neuroimage 54, 1854-1861. doi: 10.1016/j.neuroimage.2010.09.061

Faria, A. V., Zhang, J., Oishi, K., Li, X., Jiang, H., Akhter, K., et al. (2010). Atlas-based analysis of neurodevelopment from infancy to adulthood using diffusion tensor imaging and applications for automated abnormality detection. Neuroimage 52, 415-428. doi: 10.1016/j.neuroimage.2010.04.238

Farrell, J. A., Landman, B. A., Jones, C. K., Smith, S. A., Prince, J. L., van Zijl, P. C., et al. (2007). Effects of signal-to-noise ratio on the accuracy and reproducibility of diffusion tensor imaging-derived fractional anisotropy, mean diffusivity, and principal eigenvector measurements at $1.5 \mathrm{~T}$. JMRI $26,756-767$. doi: $10.1002 /$ jmri.21053
Farrell, J. A., Smith, S. A., GordonLipkin, E. M., Reich, D. S., Calabresi, P. A., and van Zijl, P. C. (2008). High b-value q-space diffusionweighted MRI of the human cervical spinal cord in vivo: feasibility and application to multiple sclerosis. Magn. Reson. Med. 59, 1079-1089. doi: $10.1002 / \mathrm{mrm} .21563$

Farrell, J. A., Zhang, J., Jones, M. V., Deboy, C. A., Hoffman, P. N., Landman, B. A., et al. (2010). Q-space and conventional diffusion imaging of axon and myelin damage in the rat spinal cord after axotomy. Magn. Reson. Med. 63, 1323-1335. doi: 10.1002/mrm.22389

Florence, S. L., Taub, H. B., and Kaas, J. H. (1998). Large-scale sprouting of cortical connections after peripheral injury in adult macaque monkeys. Science (N.Y.) 282, 1117-1121. doi: 10.1126/science.282.5391.1117

Fountas, K. N., Kapsalaki, E. Z., Jackson, J., Vogel, R. L., and Robinson, J. S. Jr. (1998). Cervical spinal cordsmaller than considered? Spine 23, 1513-1516. doi: 10.1097/00007632199807150-00001

Freund, P., Weiskopf, N., Ward, N. S., Hutton, C., Gall, A., Ciccarelli, O., et al. (2011). Disability, atrophy and cortical reorganization following spinal cord injury. Brain 134, 1610-1622. doi: 10.1093/brain/awr093

Friston, K. J., Holmes, A. P., Worsley, K. J., Poline, J. P., Frith, C. D., and Frackowiak, R. S. J. (1994). Statistical parametric maps in functional imaging: a general linear approach. Hum. Brain Mapp. 2, 189-210. doi: 10.1002/hbm.460020402

Gilmore, C. P., DeLuca, G. C., Bo, L., Owens, T., Lowe, J., Esiri, et al. (2005). Spinal cord atrophy in multiple sclerosis caused by white matter volume loss. Arch. Neurol. 62, 1859-1862. doi: 10.1001/archneur.62.12.1859

Henderson, L. A., Gustin, S. M., Macey, P. M., Wrigley, P. J., and Siddall, P. J. (2011). Functional reorganization of the brain in humans following spinal cord injury: evidence for underlying changes in cortical anatomy. $J$. Neurosci. 31, 2630-2637. doi: 10.1523/JNEUROSCI.2717-10.2011

Himberg, J., Hyvarinen, A., and Esposito, F. (2004). Validating the independent components of neuroimaging time series via clustering and visualization. Neuroimage 22, 1214-1222. doi: 10.1016/j.neuroimage.2004.03.027

Jenkinson, M., Bannister, P., Brady, M., and Smith, S. (2002). Improved optimization for the robust and accurate linear registration and motion correction of brain images. Neuroimage 17, 825-841. doi: 10.1006/nimg.2002.1132

Jiang, H., van Zijl, P. C., Kim, J., Pearlson, G. D., and Mori, S. (2006). DtiStudio: resource program for diffusion tensor computation and fiber bundle tracking. Comput. Methods Progr. Biomed. 81, 106-116. doi: 10.1016/j.cmpb.2005.08.004

Joel, S. E., Caffo, B. S., van Zijl, P. C., and Pekar, J. J. (2011). On the relationship between seed-based and ICA-based measures of functional connectivity. Magn. Reson. Med. 66, 644-657. doi: 10.1002/mrm.22818

Jurkiewicz, M. T., Crawley, A. P., Verrier, M. C., Fehlings, M. G., and Mikulis, D. J. (2006). Somatosensory cortical atrophy after spinal cord injury: a voxel-based morphometry study. Neurology 66, 762-764. doi: $10.1212 / 01 . w n l .0000201276$. 28141.40

Jurkiewicz, M. T., Mikulis, D. J., Fehlings, M. G., and Verrier, M. C. (2010). Sensorimotor cortical activation in patients with cervical spinal cord injury with persisting paralysis. Neurorehabil. Neural Repair 24, 136-140. doi: $10.1177 / 1545968309347680$

Jurkiewicz, M. T., Mikulis, D. J., McIlroy, W. E., Fehlings, M. G., and Verrier, M. C. (2007). Sensorimotor cortical plasticity during recovery following spinal cord injury: a longitudinal fMRI study. Neurorehabil. Neural Repair 21, 527-538. doi: 10.1177/1545968307301872

Kalra, L., Perez, I., Gupta, S., and Wittink, M. (1997). The influence of visual neglect on stroke rehabilitation. Stroke 28, 1386-1391. doi: 10.1161/01.STR.28.7.1386

Kameyama, T., Hashizume, Y., and Sobue, G. (1996). Morphologic features of the normal human cadaveric spinal cord. Spine 21, 1285-1290. doi: 10.1097/00007632199606010-00001

Kirshblum, S., Millis, S., McKinley, W., and Tulsky, D. (2004). Late neurologic recovery after traumatic spinal cord injury. Arch. Phys. Med. Rehab. 85, 1811-1817. doi: 10.1016/j.apmr.2004.03.015

Klawiter, E. C., Schmidt, R. E., Trinkaus, K., Liang, H. F., Budde, M. D., Naismith, R. T., et al. (2011). Radial diffusivity predicts demyelination in ex vivo multiple sclerosis spinal cords. Neuroimage 55, 1454-1460. doi: 10.1016/j.neuroimage.2011.01.007
Ko, H. Y., Park, J. H., Shin, Y. B., and Baek, S. Y. (2004). Gross quantitative measurements of spinal cord segments in human. Spinal Cord 42, 35-40. doi: 10.1038/sj.sc.3101538

Landman, B. A., Farrell, J. A., Jones, C. K., Smith, S. A., Prince, J. L., and Mori, S. (2007). Effects of diffusion weighting schemes on the reproducibility of DTIderived fractional anisotropy, mean diffusivity, and principal eigenvector measurements at $1.5 \mathrm{~T}$. Neuroimage 36, 1123-1138. doi: 10.1016/j.neuroimage.2007.02.056

Landman, B. A., Farrell, J. A., Smith, S. A., Reich, D. S., Calabresi, P. A., and van Zijl, P. C. (2010). Complex geometric models of diffusion and relaxation in healthy and damaged white matter. NMR Biomed. 23, 152-162. doi: $10.1002 / \mathrm{nbm} .1437$

Landman, B. A., Huang, A. J., Gifford, A., Vikram, D. S., Lim, I. A., Farrell, J. A., et al. (2011). Multi-parametric neuroimaging reproducibility: A 3-T resource study. Neuroimage 54, 2854-2866. doi: 10.1016/j.neuroimage.2010.11.047

Levesque, I. R., Giacomini, P. S., Narayanan, S., Ribeiro, L. T., Sled, J. G., Arnold, D. L., et al. (2010). Quantitative magnetization transfer and myelin water imaging of the evolution of acute multiple sclerosis lesions. Magn. Reson. Med. 63, 633-640. doi: 10.1002/mrm.22244

Li, Y. O., Adali, T., and Calhoun, V. D. (2007). Estimating the number of independent components for functional magnetic resonance imaging data. Hum. Brain Mapp. 28, 1251-1266. doi: 10.1002/hbm.20359

Loy, D. N., Kim, J. H., Xie, M., Schmidt, R. E., Trinkaus, K., and Song, S. K. (2007). Diffusion tensor imaging predicts hyperacute spinal cord injury severity. J. Neurotr. 24, 979-990. doi: 10.1089/neu.2006.0253

Lundell, H., Barthelemy, D., Skimminge, A., Dyrby, T. B., Biering-Sorensen, F., and Nielsen, J. B. (2011). Independent spinal cord atrophy measures correlate to motor and sensory deficits in individuals with spinal cord injury. Spinal Cord 49, 70-75. doi: 10.1038/sc. 2010.87

Maes, F., Vandermeulen, D., and Suetens, P. (1999). Comparative evaluation of multiresolution optimization strategies for multimodality image registration by maximization of mutual information. Med. Image Anal. 3, 373-386. doi: 10.1016/S1361-8415(99)80030-9 
Maynard, F. M. Jr., Bracken, M. B., Creasey, G., Ditunno, J. F. Jr., Donovan, W. H., Ducker, T. B., et al. (1997). International standards for neurological and functional classification of spinal cord injury. american spinal injury association. Spinal Cord 35, 266-274. doi: 10.1038/sj.sc.3100432

McDonald, J. W., and Sadowsky, C. (2002). Spinal-cord injury. Lancet 359, 417-425. doi: 10.1016/S01406736(02)07603-1

Naismith, R. T., Xu, J., Tutlam, N. T., Snyder, A., Benzinger, T., Shimony, J., et al. (2009). Disability in optic neuritis correlates with diffusion tensor-derived directional diffusivities. Neurology 72, 589-594. doi: $\quad 10.1212 / 01 . w n l .0000335766$. 22758.cd

Nathan, P. W. (1994). Effects on movement of surgical incisions into the human spinal cord. Brain 117(Pt 2), 337-346. doi: 10.1093/brain/117.2.337

Oishi, K., Mori, S., Donohue, P. K., Ernst, T., Anderson, L., Buchthal, S., et al. (2011). Multi-contrast human neonatal brain atlas: application to normal neonate development analysis. Neuroimage 56, 8-20. doi: 10.1016/j.neuroimage.2011.01.051

Oishi, K., Zilles, K., Amunts, K., Faria, A., Jiang, H., Li, X., et al. (2008). Human brain white matter atlas: identification and assignment of common anatomical structures in superficial white matter. Neuroimage 43, 447-457. doi: 10.1016/j.neuroimage.2008.07.009

Schmithorst, V. J., and Holland, S. K. (2004). Comparison of three methods for generating group statistical inferences from independent component analysis of functional magnetic resonance imaging data. JMRI 19, 365-368. doi: 10.1002/jmri.20009
Seeley, W. W., Menon, V., Schatzberg, A. F., Keller, J., Glover, G. H., Kenna, H., et al. (2007). Dissociable intrinsic connectivity networks for salience processing and executive control. J. Neurosci. 27, 2349-2356. doi: 10.1523/JNEUROSCI.5587-06.2007

Seitz, R. J., Knorr, U., Azari, N. P., Herzog, H., and Freund, H. J. (1999). Visual network activation in recovery from sensorimotor stroke. Restor. Neurol. Neurosci. 14, 25-33.

Sigmund, E. E., Suero, G. A., Hu, C., McGorty, K., Sodickson, D. K., Wiggins, G. C., et al. (2011). High-resolution human cervical spinal cord imaging at $7 \mathrm{~T}$. NMR Biomed. 25, 891-899. doi: 10.1002/nbm.1809

Smith, S. A., Golay, X., Fatemi, A., Jones, C. K., Raymond, G. V., Moser, H. W., et al. (2005). Magnetization transfer weighted imaging in the upper cervical spinal cord using cerebrospinal fluid as intersubject normalization reference (MTCSF imaging). Magn. Reson. Med. 54, 201-206. doi: 10.1002/ mrm.20553

Smith, S. A., Jones, C. K., Gifford, A., Belegu, V., Chodkowski, B., Farrell, J. A., et al. (2010). Reproducibility of tract-specific magnetization transfer and diffusion tensor imaging in the cervical spinal cord at 3 tesla. NMR Biomed. 23, 207-217. 10.1002/nbm. 1447

Song, S. K., Sun, S. W., Ramsbottom, M. J., Chang, C., Russell, J., and Cross, A. H. (2002). Dysmyelination revealed through MRI as increased radial (but unchanged axial) diffusion of water. Neuroimage 17, 1429-1436. doi: 10.1006/nimg.2002.1267

Song, S. K., Yoshino, J., Le, T. Q., Lin, S. J., Sun, S. W., Cross, A. H., et al. (2005). Demyelination increases radial diffusivity in corpus callosum of mouse brain. Neuroimage 26, 132-140. doi: 10.1016/j.neuroimage.2005.01.028

Stevens, M. C. (2009). The developmental cognitive neuroscience of functional connectivity. Brain Cogn. 70, 1-12. doi: 10.1016/j.bandc.2008.12.009

Vazquez, X. M., Rodriguez, M. S., Penaranda, J. M., Concheiro, L. and Barus, J. I. (2008). Determining prognosis after spinal cord injury. J. Foren. Legal Med. 15, 20-23. doi: 10.1016/j.jflm.2007.06.003

White, L. E., Andrews, T. J., Hulette, C. Richards, A., Groelle, M., Paydarfar, J., et al. (1997). Structure of the human sensorimotor system. II: lateral symmetry. Cereb. Cortex (N.Y) 7, 31-47.

Wolff, S. D., and Balaban, R. S. (1989). Magnetization transfer contrast (MTC) and tissue water proton relaxation in vivo. Magn. Reson. Med. 10, 135-144. doi: 10.1002/mrm. 1910100113

Woods, R. P., Grafton, S. T., Holmes, C. J., Cherry, S. R., and Mazziotta, J. C. (1998). Automated image registration: I. general methods and intrasubject, intramodality validation. $J$. Comput. Ass. Tomogr. 22, 139-152. doi: 10.1097/00004728-19980100000027

Wrigley, P. J., Gustin, S. M., Macey, P. M., Nash, P. G., Gandevia, S. C., Macefield, V. G., et al. (2009). Anatomical changes in human motor cortex and motor pathways following complete thoracic spinal cord injury. Cereb. Cortex (N.Y) 19, 224-232.

Zackowski, K. M., Smith, S. A., Reich, D. S., Gordon-Lipkin, E., Chodkowski, B. A., Sambandan, D. R., et al. (2009). Sensorimotor dysfunction in multiple sclerosis and column-specific magnetization transfer-imaging abnormalities in the spinal cord. Brain 132, 1200-1209. doi: 10.1093/brain/awp032

Conflict of Interest Statement: Dr. Pekar serves as Manager of the F. M. Kirby Research Center for Functional Brain Imaging, which receives research support from Philips HealthCare, which manufactures the MRI scanners used in this study. Dr. van Zijl has grant funding from Philips Healthcare. In addition, Dr. van Zijl is the inventor of technology that is licensed to Philips and is a paid lecturer for Philips. This arrangement has been approved by Johns Hopkins University in accordance with its conflict of interest policies. The other authors declare that the research was conducted in the absence of any commercial or financial relationships that could be construed as a potential conflict of interest.

Received: 25 February 2013; accepted: 03 June 2013; published online: 25 June 2013.

Citation: Choe AS, Belegu V, Yoshida S, Joel S, Sadowsky CL, Smith SA, van Zijl PCM, Pekar JJ and McDonald JW (2013) Extensive neurological recovery from a complete spinal cord injury: a case report and hypothesis on the role of cortical plasticity. Front. Hum. Neurosci. 7:290. doi: 10.3389/fnhum.2013.00290

Copyright (c) 2013 Choe, Belegu, Yoshida, Joel, Sadowsky, Smith, van Zijl, Pekar and McDonald. This is an openaccess article distributed under the terms of the Creative Commons Attribution License, which permits use, distribution and reproduction in other forums, provided the original authors and source are credited and subject to any copyright notices concerning any third-party graphics etc. 